\title{
Sub-cycle Manipulation of Electrons in a Tunnel Junction with Phase-controlled Single-cycle THz Near-fields
}

\author{
Katsumasa Yoshioka ${ }^{1}$, Ikufumi Katayama ${ }^{1}$, Yusuke Arashida ${ }^{1}$, Atsuhiko Ban ${ }^{1}$, \\ Yoichi Kawada ${ }^{2}$, Hironori Takahashi ${ }^{2}$, and Jun Takeda ${ }^{1, *}$ \\ ${ }^{1}$ Department of Physics, Yokohama National University, Yokohama 240-8501, Japan \\ ${ }^{2}$ Central Research Laboratory, Hamamatsu Photonics K. K., Hamamatsu 434-8601, Japan
}

\begin{abstract}
By utilizing terahertz scanning tunneling microscopy (THz-STM) with a carrier envelope phase shifter for broadband $\mathrm{THz}$ pulses, we could successfully control the near-field-mediated electron dynamics in a tunnel junction with sub-cycle precision. Measurements of the phase-resolved sub-cycle electron tunneling dynamics revealed an unexpected large carrier-envelope phase shift between far-field and near-field single-cycle THz waveforms.
\end{abstract}

\section{Introduction}

Recent progress on coherent manipulation of electrons under a non-perturbative regime with the carrier-envelope phase (CEP) locked ultrashort pulses holds great promise for overcoming the bandwidth limitation of modern electronics [1]. The development of this lightwave electronics has been stimulated by locally enhanced near-field in nanostructures, and the optical near-field in nanoscale metal tip can be precisely characterized via streaking spectroscopy [2,3]. Recently, we observed that the electron tunneling through metallic nanogaps can be accelerated by single-cycle terahertz $(\mathrm{THz})$ electric fields in percolated $\mathrm{Au}$ nanostructures [4] and in a single tunnel junction between metal nanoelectrodes [5]. By developing terahertz scanning tunneling microscope (THz-STM) in ultrahigh vacuum, coherent manipulation of electrons was achieved even at the atomic scale [6,7]. However, since the first development of THz-STM [8], an experimental method for retrieving $\mathrm{THz}$ near-fields in a tunnel junction remains elusive. Therefore, the characterization and active control of $\mathrm{THz}$ near-fields in a tunnel junction is indispensable for advancing ultrafast nanoscale manipulation of electrons.

In the present work, using THz-STM coupled with a CEP shifter for broadband $\mathrm{THz}$ pulses [9], we demonstrate that desirable phase-controlled $\mathrm{THz}$ near-fields can be produced in a tunnel junction [10]. Measurements of the phase-resolved sub-cycle electron tunneling dynamics revealed a large CEP shift between the THz far- and near-field waveforms. Based on an antenna-model that assigns an equivalent RLC circuit to the nanotip [11], we could retrieve the $\mathrm{THz}$ near-field in a tunnel junction. By utilizing the phase-controlled double

\footnotetext{
* Corresponding author: jun@ynu.ac.jp
} 
$\mathrm{THz}$ pulses with a given time interval, we could successfully manipulate the timing and direction of electron tunneling over the femtosecond timescale.

\section{Figures and tables}

Figure 1(a) illustrates the basic concept of our CEP-controlled THz-STM. We used highly oriented pyrolytic graphite (HOPG) as a typical sample because of its atomically flat surface. Single-cycle $\mathrm{THz}$ electric fields were generated by optical rectification of nearinfrared femtosecond pulses in a LiNbO3 prism with a tilted-pulse-front configuration. Subsequently, the generated $\mathrm{THz}$ pulses were guided into a CEP shifter that consists of three optical elements: a quarter-wave plate (QWP), half-wave plate (HWP), and wire grid polarizer (WGP) [9]. The $\mathrm{THz}$ pulses were guided into one of two paths by tuning a removable gold-coated mirror: one was used for characterizing the $\mathrm{THz}$ waveforms by electro-optic sampling (EOS) and the other was used for delivering the $\mathrm{THz}$ pulses to a tunnel junction of the STM. Figures 1(b) and 1(c) show the far-field single-cycle $\mathrm{THz}$ waveforms with different CEPs measured via the EOS and their Fourier-transformed spectra, respectively. As shown in Figs. 1(b) and 1(c), the CEP value can be continuously tuned from $0-2 \pi$ without any variations in the time delay, pulse duration, and frequency component.

\section{Results and discussion}

Figure 2(a) shows the number of rectified electrons as a function of CEP together with those calculated using the Simmons model [12] for the corresponding incident $\mathrm{THz}$ electric fields. The image of $\mathrm{Pt} / \mathrm{Ir}$ nanotip used in this experiment is also shown in the inset. The direction and number of electron tunneling through the junction are strongly dependent on the CEP; the number of rectified electrons is maximized at $0.67 \pi$ for electrons tunneling from the nanotip to the sample, while at $1.67 \pi$ for the opposite scenario. In these cases, the $\mathrm{THz}$ waveform in the junction is expected to be a single-cycle co-sinusoidal near-field. On the other hand, the number of rectified electrons is minimized at $0.07 \pi, 1.12 \pi$ and $2.06 \pi$, corresponding to a sinusoidal near-field in the junction. Note that there is a measurable CEP shift between the calculated result of far-field and the experimental result of the near-field. This deviation can be attributed to the change of the local-field at the apex of a nanotip induced by the antenna structure [11]. By assigning an equivalent RLC circuit to the antenna response of the nanotip, the rectified electrons mediated via the $\mathrm{THz}$ near-field in the junction are reasonably retrieved as shown by the red circles. Finally, in order to investigate ultrafast dynamics of $\mathrm{THz}$ near-field-mediated electron tunneling, we employed a dual-phase double-pulse scheme with delay- and CEP-controlled THz pulses. As shown 
in Figs. 2(b), the bidirectional sub-picosecond electron burst was generated by adjusting the CEP-controlled pulse to yield a sinusoidal near-field in the junction. Furthermore, we found that the timing and direction of ultrafast electron burst can be manipulated over the femtosecond timescale by actively control the $\mathrm{THz}$ near-field with precisely adjusting the CEP of CEP-controlled pulse [10].
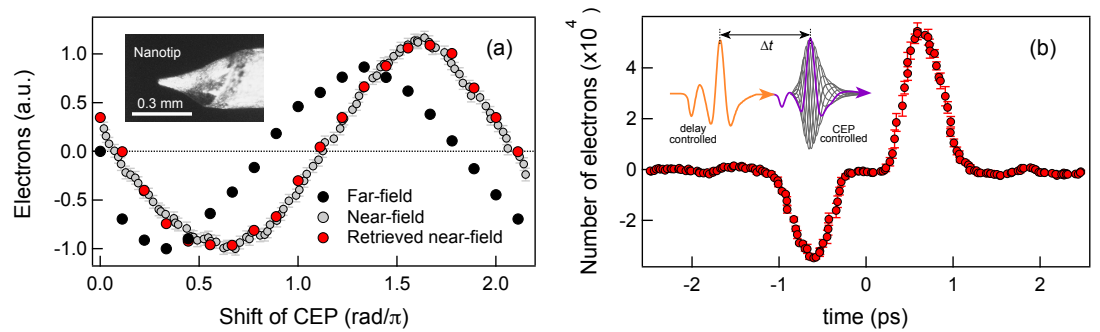

Fig. 2 (a) CEP dependence of rectified electrons induced by single-cycle terahertz pulses (grey circles). Black and red circles are those calculated for THz far-field obtained with EOS and nearfield retrieved using an antenna-model that assigns an equivalent RLC circuit to the nanotip, respectively. The inset shows optical micrograph of Pt/Ir nanotip. (b) Number of electrons as a function of delay time between the sinusoidal near-field and the delay-controlled $\mathrm{THz}$ waveform. The inset shows schematic of the experimental concept.

\section{References}

1. F. Krausz, M.I. Stockman, Nat. Photonics 8, 205 (2014)

2. L. Wimmer et al., Nat. Phys. 10, 432 (2014)

3. B. Förg et al., Nat. Commun. 7, 11717 (2016)

4. K. Yoshioka et al., Nano Lett. 15, 1036 (2015)

5. K. Yoshioka et al., Nat. Photon. 10, 762 (2016)

6. T. L. Cocker et al., Nature 539, 263 (2016)

7. V. Jelic et al., Nat. Phys. 13, 591 (2017)

8. T. L. Cocker et al., Nat. Photon. 7, 620 (2013)

9. Y. Kawada, T. Yasuda, and H. Takahashi, Opt. Lett. 41, 986 (2016)

10. K. Yoshioka et al., Nano Lett. Article ASAP, DOI: 10.1021/acs.nanolett.8b02161

11. K. Wang et al., Appl. Phys. Lett. 85, 2715 (2004)

12. J. G. Simmons, J. Appl. Phys. 34, 1793 (1963) 